\title{
A Matched Case-Control Study to Identify Risk Determinants of Tuberculosis in Bangladesh
}

\author{
Malabika Sarker, Fahmida Homayra, A. H. M. Mahbub Latif, Mrittika Barua, Avijit Saha, Sukanta Paul, Rahima \\ Akter, Shayela Islam, Akramul Islam \\ BRAC University, Bangladesh
}

\section{Introduction}

According to the estimates of 2015, the global incidence rate for tuberculosis (TB) is 142 per 100,000 population [1]. In Bangladesh, the prevalence rate of TB is 382 per 100,000 population and incidence rate is 225 per 100,000 population [1], [2]. Over the years researchers primarily focused on socioeconomic, cultural, and biological factors as the determinants of causation of TB. According to Hargreaves et al. (2011), global socio- economic inequalities, high population mobility, and rapid urbanization influenced key social determinants of TB such as malnutrition, food insecurity, poor environmental conditions, financial, cultural, and geographical factors [3]. These social determinants, in turn, affect the four stages pathogenesis of TB: exposure to infection, progression to disease, diagnosis and treatment, adherence, and success. According to WHO 2016 report, TB epidemic is more severe than it was previously estimated [1]. As developing countries in Asia and Africa are carrying major TB burden [1] and often face difficulties in accessing medical care, social and economic determinants of TB must be appropriately addressed together with the adequate implementation of the TB prevention programs.

Various studies delved into varieties of factors that can determine the occurrence of
TB. Factors like lower BMI, less access to running water, bathroom or flushing toilet, smoking, alcohol use [4] and TB contact [5] were found to be associated with the occurrence of TB. Although the matched case-control study conducted by Ladefoged et al. in 2011 in Greenland didn't find diabetes mellitus (DM) to be associated with TB, DM repeatedly came up in other studies as an important risk factor to TB [6]-[8]. Additionally, evidence was found for indoor air pollution [7], crowding, and poor ventilation [5], [9] to be associated with TB.

In Bangladesh, there was hardly any study conducted on identifying risk factors for TB. In 2013, Flora et al. considered an unmatched case-control study to assess the risk factors for multi-drug resistant TB in peri-urban areas [9]. It was found that age less than 40 years and $\mathrm{TB}$ contact to be the strongest determinants of multi-drug resistant TB but not educational status, occupation, monthly family income, and housing status.

However, the results of this study must be dealt with caution as it was an unmatched case-control study conducted in peri- urban areas, with cases who were TB patients undergoing treatment and controls were cured TB patients. It is mandatory to note that urban slums in Dhaka, Bangladesh have revealed to have a higher prevalence of sputum-positive TB cases than the overall 
prevalence of the urban setting [9] and it is in these slums where people usually live in overcrowded surroundings with poorly ventilated rooms [9]. Therefore there might be a high possibility of housing status to be highly associated with TB in general in urban Bangladesh. Also, none of these studies provide any information regarding roles of such factors in rural context. Therefore, the current study aimed to deduce determinants of TB in Bangladesh, both in urban and rural areas. We specifically explored whether education, socio- economic condition, personal health and lifestyle factors such as a history of TB or TB-DM co-occurrence, smoking habits, living in a crowded environment are associated with TB incident in Bangladesh. Despite the fact that the National TB Control Programme provides cost-free treatment all over the country Bangladesh did not meet the TB-related goals of Millennium Development Goals 2015 [1]. Moreover, Bangladesh is one of the thirty major TB burden countries in the world [1]. Therefore, there has been a need for research on TB risk determinants in Bangladesh to implement effective interventions as part of the national effort of TB prevention and control.

\section{Materials and methods Ethics considerations}

Ethical approval was taken from Ethical Review Board of BRAC Institute of Global Health, BRAC University. An authorization letter was issued by BRAC TB Control Programme to access the TB register of the program. The purpose of the study and the individual's rights as respondent was clearly read out to the study participants. Verbal consent was taken from the respondents who were informed regarding the voluntary nature of participation. Their names were not recorded anywhere and their information were kept confidential. Interviews were conducted in a private setting to preserve the privacy of the respondents for sensitive questions.

\section{Study population and design}

A case-control study was carried out with TB patients and matched controls in Bangladesh. Six divisions of Bangladesh, namely, Dhaka, Chittagong, Sylhet, Barisal, Khulna, and Rangpur were selected as study areas. In each division, one urban and one rural area were selected. Two healthy controls were matched with per case according to sex and age (no more than five-year age difference). TB cases were randomly selected from BRAC TB Control Programme and two matched controls were chosen from the community where the TB case belonged. A total of 360 cases were matched with 720 controls. The study was designed to have $80 \%$ power,

0.05 significance level, a ratio of case to control 1:2 and odds ratio of 1.5 [10]. Study participants aged at least 18 years who gave their informed verbal consent were included in the study. We used a structured questionnaire to gather information on age, educational status, marital status, occupation, housing status, socio-economic status (SES), malnutrition, history of TB, peer history of $\mathrm{TB}$, diabetes status, diabetes history in the family, smoking habits, substance use habits, etc.

\section{Data collection}

BRAC TB Control Programme maintains TB patient register and assigns a unique $\mathrm{TB}$ registration number to each patient as a routine practice. The patient register was used as the sampling frame to select cases. 
The matched controls were selected with the help of BRAC community health workers, who were known as Sastho-Karmi (SK) and Sastho- Sebika (SS). SSs are a group of female workers who provide voluntary community health services and are recruited and trained by BRAC. SKs are salaried female supervisors of the SSs. As there was no sampling frame for controls SSs and SKs knowledge about the community was leveraged to identify the controls matched by age and sex of the selected cases.

Trained investigators interviewed the study participants in person using a pretested questionnaire. The interview was conducted in the local language. Quality assurance was conducted in two phases; in phase I the investigator reviewed the collected data, and in phase II a supervisor double checked the data quality. Data entry and data cleaning were done using STATA 13 [11].

\section{Variables}

Potential risk factor variables were grouped into demographic, socio-economic, and personal health-related categories. Demographic variables include the area of residence (urban/rural), marital status, educational status, employment status, and occupation. Socio-economic variables include monthly income, building materials of the main parts of the house (e.g. floor, roof, and wall), the number of rooms for sleeping, the source of drinking water, the types of toilet used, the types of fuel used for cooking, landownership, the number of domestic animals (e.g. cow, goat, buffalo, hen), electric power supply, possession of electronic goods (e.g. radio, TV, etc.), and possession of vehicles (e.g. rickshaw, bicycle, motor-bike). Personal health-related variables include malnutrition, diabetes status, previous history of TB, family history of TB, the habit of smoking cigarettes, the habit of using tobacco other than cigarettes, the number of people sleeping in the respondent's room, and unhygienic surroundings.

A principal component analysis (PCA) was done to construct asset index. Socioeconomic variables such as the building materials of the house, the number of rooms for sleeping, the source of drinking water, the types of toilet used, the types of fuel used for cooking, landownership, the number of domestic animals, electric power supply, possession of electronic goods, and possession of vehicles were used to conduct the PCA. The first principal component was predicted from the fitted model to estimate the asset index. The quintiles of the asset index were considered as the determinant of the SES [12], higher quintiles are indicators of a higher SES.

\section{Statistical analysis}

The distributions of different risk factors were explored for cases and controls separately. A conditional logistic regression (CLR) model [12] was considered to identify the risk factors for TB. The effects of different risk factors were measured by adjusted odds ratios (aOR) and the corresponding 95\% confidence intervals (CIs). We fitted a CLR model with the quintiles of the asset index, household type, the area of residence, marital status, educational status, occupational status, the number of people sleeping in the respondent's room, diabetes status, family history of diabetes, previous history of TB, family history of TB, the habit of smoking cigarettes, and the habit of tobacco use other 
than cigarettes. The variables which were statistically significant ( $\mathrm{p}-$ value $<0.05$ ) were kept in the final model. The area of residence (urban/rural) was dropped from the model because the exposure status was same among the matched pairs. It is important to note that, those matched pairs do not contribute in estimating odds ratio [12]. The PCA was done using the pca command, and the CLR analysis was done using the clogit command in STATA 13 [11] Results

\section{Demographic factors}

The analysis was completed on 360 cases and 720 controls. Age of the cases varied from 18 to 90 years with a median of 40 years, $56 \%$ of them were males and 52\% lived in the urban area. Age of the controls varied from 18-92 years with a median of 38 years, and the percentage distribution of gender and the area of residence (rural/urban) were similar as that of the cases. Most of the cases, $41 \%$, had no education, $27 \%$ had primary education, $28 \%$ had secondary education, and only $4 \%$ had the higher secondary education. The distribution of level of education among controls was not very different than that of the cases. Most of the controls, 36\%, had secondary education, $32 \%$ had no education, $27 \%$ had primary education, and only $5 \%$ had the higher secondary education. Among the cases $78 \%$ were married, and among the controls $79 \%$ were married. The distribution of occupational status was similar in cases and controls. Among the cases 45\% were unemployed, $42 \%$ were employed, and 13\% were retired. Among the controls 43\% were unemployed, 54\% were employed, and 3\% were retired. (TABLE I)

\section{Socio-economic factors}

The socio-economic distribution of cases and controls were similar. Approximately, 70\% of the cases and controls were in the upper level of SES. Average monthly income of the cases and controls were 10000 BDT; although there were few respondents' with monthly income over 50000

BDT. Additionally, the type of household of the cases and controls were similar, 83\% cases and

89\% controls lived in imperfect houses (house made of mud or wood) or in semibuilding houses.

\section{(TABLE I)}

\section{Personal health-related factors}

Approximately, $7 \%$ of the cases and 3\% of the controls had a history of TB whereas $43 \%$ cases and $46 \%$ controls had a history of TB in the family. The percentage of patients with diabetes was low in both groups, among the cases $12 \%$ compared to $7 \%$ among the controls. Approximately, $72 \%$ of the cases and $71 \%$ of the controls never smoked a cigarette, $7 \%$ of the cases and $25 \%$ of the controls were current smokers, and $21 \%$ of the cases and $5 \%$ of the controls were former smokers. (TABLE I) 
Table I. Descriptive statistics of demographic, socio- economic, and health-related factors for cases and controls

\begin{tabular}{|c|c|c|}
\hline Characteristics and category & Cases $(\mathrm{N}=360)$ count $(\%)$ & Cases $(\mathrm{N}=720)$ count $(\%)$ \\
\hline $\begin{array}{l}\text { Demographic factors Age, } \\
\text { median (range) }\end{array}$ & $40(18-90)$ & $38(18-92)$ \\
\hline Sex & & \\
\hline Male & $176(56)$ & $351(56)$ \\
\hline Female & $139(44)$ & $279(44)$ \\
\hline Area Urban & $164(52)$ & 328 (52) \\
\hline $\begin{array}{l}\text { Rural } \\
\text { Marital status }\end{array}$ & $151(48)$ & 302 (48) \\
\hline Single & $44(14)$ & $90(14)$ \\
\hline Married & 247 (78) & 495 (79) \\
\hline Divorced & $2(0.5)$ & 0 \\
\hline Separated & $5(0.95)$ & $3(0.5)$ \\
\hline Widowed & $19(6)$ & $42(6.5)$ \\
\hline Education No & & \\
\hline education & $128(41)$ & $204(32)$ \\
\hline Primary & $85(27)$ & $168(27)$ \\
\hline Secondary & $88(28)$ & $228(36)$ \\
\hline Higher & $14(4)$ & $30(5)$ \\
\hline Occupational status & & \\
\hline Employed & $133(42)$ & 339 (54) \\
\hline Unemployed & $141(45)$ & $270(43)$ \\
\hline Retired & $41(13)$ & $21(3)$ \\
\hline Socio-economic factors & & \\
\hline Asset index quintile & & \\
\hline 1 & $13(4)$ & $19(3)$ \\
\hline 2 & $29(9)$ & $57(9)$ \\
\hline 3 & $53(17)$ & $118(18)$ \\
\hline 4 & $122(38)$ & 252 (39) \\
\hline 5 & $104(33)$ & $196(31)$ \\
\hline Monthly income (BDT), & & \\
\hline Median & 10000 & 10000 \\
\hline Type of household & & \\
\hline Imperfect/ semi-building & $256(83)$ & $572(89)$ \\
\hline Building & $56(17)$ & $70(11)$ \\
\hline Personal health factors & & \\
\hline Previous history of TB & & \\
\hline $\begin{array}{l}\text { Yes } \\
\text { No }\end{array}$ & $21(7)$ & $20(3)$ \\
\hline Peer history of TB & $299(93)$ & $615(97)$ \\
\hline Yes & 135 (43) & $288(46)$ \\
\hline No & $163(52)$ & $292(47)$ \\
\hline Do not know & $15(5)$ & $44(7)$ \\
\hline Malnutrition & & \\
\hline
\end{tabular}




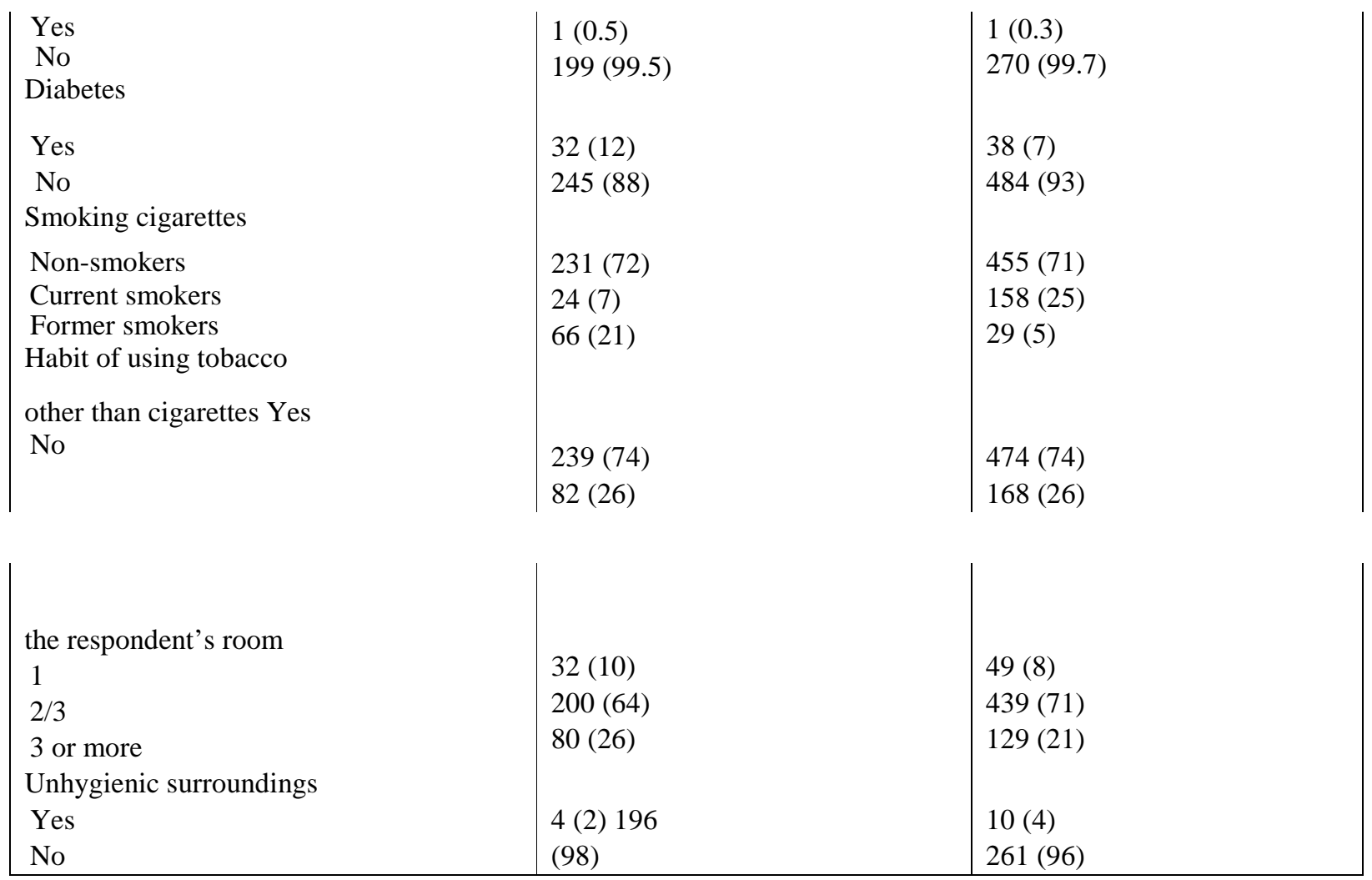

Number of people living in

\section{Conditional logistic regression}

In the CLR model, the aOR of four risk factors were found statistically significant. Namely, the level of education, the types of household, previous history of TB, and the habit of smoking cigarettes. As socioeconomic factor was of primary interest, the quintile of asset index was retained in the final model. (TABLE II)

\section{Table II. Conditional logistic regression model for TB risk factors}

\begin{tabular}{|l|l|l|l|l|}
\hline Risk factor & aOR* & Standard error & p-value & $95 \%$ CI \\
\hline Level of education & & & & \\
No education & 1 & & & \\
Primary & 0.80 & 0.17 & 0.28 & $0.52-1.2$ \\
Secondary or more & 0.5 & 0.11 & 0.002 & $0.32-0.778$ \\
Asset quintile index 1 & & & & \\
& 1 & & & \\
2 & 0.62 & 0.31 & 0.34 & $0.23-1.64$ \\
3 & 0.53 & 0.25 & 0.18 & $0.2-1.35$
\end{tabular}




\begin{tabular}{|l|l|l|l|l|}
4 & 0.70 & 0.34 & 0.47 & $0.27-1.82$ \\
5 & 0.74 & 0.39 & 0.58 & $0.26-2.12$ \\
$\begin{array}{l}\text { Type of household Improper/semi- } \\
\text { building }\end{array}$ & 1 & & & \\
Building & 2.54 & 0.74 & 0.001 & $1.44-4.52$ \\
$\begin{array}{l}\text { Smoking cigarette Non- } \\
\text { smokers }\end{array}$ & 1 & & & \\
Current smokers & 0.27 & 0.08 & $<0.001$ & $0.15-0.49$ \\
Former smokers & 5.27 & 1.68 & $<0.001$ & $2.82-9.86$ \\
Previous history of TB & 1 & & & \\
No & 2.04 & 0.68 & 0.03 & $1.05-3.94$ \\
Yes & & \\
\hline
\end{tabular}

*ORs were adjusted for all covariates retained in the model

The risk of TB decreased with the increase of the level of education. The risk was $20 \%$ and $50 \%$ lower among people with primary education and secondary or higher level of education, respectively, compared to the people with no education. The aOR for the education levels were 0.80 (95\% CI: 0.52 , 1.2), 0.5 (95\% CI: 0.32, 0.78), respectively. Thus, only secondary or higher level of education reached statistical significance.

The risk of TB was 38\% lower among respondents whose SES was in the second quintile compared to the respondents whose SES was in the first quintile [aOR $=0.62,95 \%$ CI: $(0.23,1.64)]$. However, the aOR was not statistically significant. Additionally, the aOR of the third $[0.53$, 95\% CI: $(0.2,1.35)]$, fourth [0.70, 95\% CI: $(0.27,1.87)]$, and fifth [0.74, 95\% CI: $(0.26,2.12)]$ quintiles were also not statistically significant.

Among the health factors, a history of TB and smoking cigarettes were found statistically significant. The aOR of the previous history of TB was 2.04 (95\% CI: 1.05, 3.95) compared to no history of TB. Thus the risk increased 2.04 times among the respondents who had TB in the past than the respondents

whose histories were clean. The risk of TB was $72 \%$ lower among the current smokers than that of the non- smokers $[\mathrm{aOR}=0.28$, 95\% CI: $(0.16,0.49)]$. Here, the non- smokers are respondents who never smoked a cigarette. Nonetheless, the risk of TB among the former smokers was 5.27 (95\% CI: 2.82 , 9.86) times than that of the nonsmokers.

In the CLR model marital status, occupational status, the number of people sleeping in the respondent's room, diabetes status, family history of diabetes, peer history of TB, and the habit of tobacco use other than cigarettes were not statistically significant.

\section{Discussion}

This research was conducted during the transition from the era of the Millennium Development Goals (1990-2015) to the Sustainable Development Goals (20162030). Accordingly, the WHO marked the changeover from the stop-TB strategy to the end-TB strategy. The target of the stopTB strategy was to reverse the TB epidemic whereas the targets of the end-TB strategy are to reduce $80 \%$ of TB incidence rate and $90 \%$ of TB deaths by 2030 compared to 2015 [1]. To meet the TB-related goals implementation of the determinant-specific intervention is 
warranted. Despite some progress in the TB treatments and new diagnostics, TB research and development of new and powerful interventions remains relatively underfunded [1]. Against this background, we studied risk determinants of $\mathrm{TB}$ in Bangladesh. In our knowledge, this is the first study in Bangladesh that covered both rural and urban areas to determine TB risk factors in Bangladesh.

The research started with potential risk factors regarding demographic, socioeconomic, and lifestyle factors. We identified a few variables as risk factors for $T B$ in Bangladesh. Among the demographic factors the level of education was associated with TB. However, there is no significant difference between the risks of developing TB among the respondents having the primary level of education and the respondents having no education. The results showed that the secondary or higher level of education was highly protective against TB. This result is in agreement with previous studies conducted in developing countries in South Asia and Africa [10], [13]. Although education is highly subsidized by the Government of Bangladesh high prevalence of school drop-outs is observed due to lack of interest, adolescent engagement in paid works, and early marriage [14]. We recommend implementing effective interventions to reduce the number of school drop- outs as means of the National TB Control Programme.

In addition, marital status was not found associated with TB in our study. However, it was found significant in a case- control study conducted in three countries in Africa. Findings showed that the risk of TB was higher in widowed/divorced/single people compared with married people. The association of economic condition with marital status in rural Africa was considered as the hidden reason behind the significant risk difference. As the widowed, divorced, or single people are financially dependent on the family it may affect their lifestyle factors (such as shared housing, low level of health care, etc.) that are independent risk factors for TB [15]. Nevertheless, in Bangladesh, the norm is to live with the family and share household income to pay living expenses including health care. That is, financial ability or lifestyle factors of all family members might be similar regardless of marital status. Also, we did not find any evidence for association between occupational status and TB. SES might play a more important role as a risk factor than individual level occupational status. Nevertheless, a similar study in rural Malawi found positive association of TB with occupational status. The results showed that the risk of TB was 1.90 times higher among the people employed in small businesses/trade/manual work [95\% CI: (1.50, 2.41)] and 1.94 times higher among the employed people in large businesses [95\% CI: $(1.46,2.59)]$ compared to the people engaged in subsistence farming [16]. Also, a study in South India showed that the prevalence of TB was higher in laborers (43.96\%) followed by white-collar workers (27\%), household workers (12\%), and unemployed or retired people (6.28\%) [17].

In most literatures, SES was identified as a risk factor for TB [2], [3], [18] [19]. We found that the risk of developing TB among the people from low SES was higher than that of the people from higher SES. Also, previous studies showed that most of the TB 
burden falls among the population with high level of poverty [2], [15], [17], [18], [20]. We recommend implementation of interventions regarding social protection to reduce poverty, the effect of financial loss or unemployment, food security, and also improvement of livelihood by cash transfer or life-skill training as means of TB prevention and control program in Bangladesh.

Among the lifestyle or personal health factors type of household, the previous history of TB, and smoking cigarettes were found significant in this study. The results showed that the risk of developing $\mathrm{TB}$ is approximately two times higher among the people with a history of TB compared to the people who were not infected before. According to the public health research report in Canada 2013, the history of active TB is one of the health factors that are associated with TB [21]. This finding is also in line with a cohort study conducted in urban Barcelona that found that TB incidence rate was 13 percent higher in the population having a history of $\mathrm{TB}$ compared to the general population [22].

Findings also showed that former smokers are highly likely to have TB compared to the people who never smoked a cigarette. It is well established in previous studies that smoking cigarette or use of tobacco increases the risk of being infected by $\mathrm{TB}$, developing active TB, and dying from it [23]-[26]. Our findings showed that current smokers are less likely to develop TB than the people who never smoked. The reason behind the unusual result could be quitting smoking upon diagnosis. In Bangladesh, tobacco control has been neglected as a mean of reduction of TB incidence. As smoking in public is practiced heavily in Bangladesh further study of the association of TB with passive smoking, especially, impact on children might help to raise awareness and strengthen tobacco control program as a mean of TB control program.

Although previously diabetes came as a risk factor in many studies [13], [27], [28], we did not find any significant association. It should be noted that the DM status was self- reported by the patients in our study. Hence, we recommend a study with proper screening method for DM status to identify the association of TB with DM in Bangladesh. We also did not find any association of developing TB with having TB or diabetes in the family. Though sharing room with too many people came as a risk factor in many studies [20], [29], [30], it was not statistically significant in our study.

Surprisingly, the results of this study showed that people living in improper houses or semibuildings were less likely to have TB than the people living in buildings. The underlying reason might be ventilation of the household and the level of air pollution in the area of residence. In this study, $62 \%$ of the improper houses or semi-buildings were in rural settings whereas $64 \%$ of the buildings were in the urban settings. The distribution of household type in the urban and rural settings and a higher risk of TB among residents in buildings indicate that there might be an association between the area of residence and TB. In Bangladesh, urban areas are more likely to be polluted than rural areas. Also, urban housings are densely populated with a poor ventilation system. This might be the secret risk factor for TB. As the controls in our study were matched from the same community where the cases belonged we did not have a sufficient number of matched pairs 
where exposure level of area of residence was different for the selected case and controls within the stratum. Thus, we could not see the association of urban/rural area with TB. Further studies are required to justify the association.

Our study is the first community-based matched case- control study in Bangladesh to identify determinants of TB. Although we explored a number of socio-demographic and health-related factors, our study has few limitations. Use of cross-sectional and selfreported data may introduce recall bias. Although it was of interest to see if unhygienic surroundings and malnutrition play any role in TB infection, our data lack the variability in this regard. Hence, a study with this sample would provide misleading results. Moreover, we could not assess the association of HIV and TB in our study. Nevertheless, Bangladesh is a low-HIV country [1]. A study involving TB-HIV patients would require a large sample.

\section{Conclusion}

This study identified few risk factors for TB in Bangladesh, namely, low level of education, lower socio-economic status, types of household, previous history of TB, and smoking cigarettes. We recommend further studies to explore the association of TB and area of residence, passive smoking, malnutrition, and DM using proper screening process. The findings of this study have important implications for the national TB prevention and control program. We recommend development of social protection, livelihood interventions, and tobacco control to strengthen the National TB Control Programme.

\section{Acknowledgment}

This work was supported by the Global Fund to Fight AIDS, Tuberculosis, and Malaria (GFATM). We received cooperation from BRAC TB Control Programme, health and nutrition population program, and BRAC. Also, we thank our research investigator team who worked very hard to collect quality data. Finally, we are thankful to our study respondents for their time and cooperation.

\section{Author's contribution}

Concept note: MB, MS, AI Investigation: AS, RA, SP, SI Methodology: MS, AHMML, AI Formal

Analysis: FH Supervision: MS, AHMML Writing-original draft: $\mathrm{FH}$ Writing-review and editing: MS, AHMML, FH

\section{References}

[1] World Health Organization, Global Tuberculosis Report 2016. Geneva: World Health Organization; 2016.

[2] Institute of Epidemiology Disease Control \& Research, Tuberculosis prevalence survey in Bangladesh 2015. Dhaka: IEDCR; 2016.

[3] J. R. Hargreaves, D. Boccia, C. A. Evans, M. Adato, M. Petticrew, and J. D. Porter, "The social determinants of tuberculosis: from evidence to action,” Am. J. Public Health, vol. 101, no. 4, 2011.

[4] K. Ladefoged, T. Rendal, T. Skifte, M. Andersson, B. Søborg, and A. Koch, "Risk factors for tuberculosis in Greenland: case-control study," Int. J. 
Tuberc. Lung Dis., vol. 15, no. 1, pp. 44-49, 2011.

[5] E. Blas, A. S. Kurup, World Health Organization, Equity, social determinants and public health programmes. Switzerland: World Health Organization; 2010.

[6] J. D. Goldhaber-Fiebert, C. Y. Jeon, T. Cohen, and M. B. Murray, "Diabetes mellitus and tuberculosis in countries with high tuberculosis burdens: individual risks and social determinants,” Int. J. Epidemiol., vol. 40, no. 2, pp. 417-428, Apr. 2011.

[7] M. Murray, O. Oxlade, and H.-H. Lin, "Modeling social, environmental and biological determinants of tuberculosis,” Int. J. Tuberc. Lung Dis., vol. 15, no. 6, pp. 64-70, Jun. 2011.

[8] V. Viswanathan et al., "Prevalence of diabetes and pre- diabetes and associated risk factors among tuberculosis patients in India,” PLoS ONE, vol. 7, no. 7, p. e41367, Jul. 2012.

[9] S. Banu et al., "Epidemiology of tuberculosis in an urban slum of Dhaka city, Bangladesh,” PLoS ONE, vol. 8, no. 10, p. e77721, Oct. 2013.

[10] N. Shetty, M. Shemko, M. Vaz, and G. D’souza, “An epidemiological evaluation of risk factors for tuberculosis in South India: a matched case-control study,” Int. J. Tuberc. Lung Dis., vol. 10, no. 1, pp. 80-86, 2006.

[11] StataCorp. 2013. Stata Statistical Software: Release 13.

College Station, TX: StataCorp LP
[12] N. E. Breslow, N. E. Day, and N. E. Breslow, The Analysis of Case-Control Studies, 8. reimpression. Lyon: International Agency for Research on Cancer, 2000.

[13] J. H. Schoeman, M. S. Westaway, and A. Neethling, "The relationship between socioeconomic factors and pulmonary tuberculosis,” Int. J. Epidemiol., vol. 20, no. 2, pp. 435-440, Jun. 1991.

[14] Sajeda Amin (ed.), Urban adolescents needs assessment survey in Bangladesh. Dhaka: BIED, BRACU, and Population Council; 2015.

[15] C. Lienhardt, "Investigation of the risk factors for tuberculosis: a case-control study in three countries in West Africa," Int. J. Epidemiol., vol. 34, no. 4, pp. 914- 923, Apr. 2005.

[16] A. Odone et al., “Association between socioeconomic position and tuberculosis in a large population-based study in rural Malawi,” PLoS ONE, vol. 8, no. 10, p. e77740, Oct. 2013.

[17] D. Gupta, K. Das, T. Balamughesh, N. Aggarwal, and $\mathrm{S}$.

K. Jindal, "Role of socio-economic factors in tuberculosis prevalence,” Indian J. Tuberc., vol. 51, no. 1, pp. 27-32, 2004.

[18] S. Jackson, A. C. Sleigh, G. J. Wang, and X. L. Liu, "Poverty and the economic effects of TB in rural China," Int. J. Tuberc. Lung Dis., vol. 10, no. 10, pp. 1104-1110, 2006.

[19] M. Muniyandi et al., "The prevalence of tuberculosis in different economic strata: a community survey from South 
India [Short Communication],” Int. J. Tuberc. Lung Dis., vol. 11, no. 9, pp. 1042-1045, 2007.

[20] S. Gupta, V. P. Shenoy, C. Mukhopadhyay, I. Bairy, and

S. Muralidharan, "Role of risk factors and socio-economic status in pulmonary tuberculosis: a search for the root cause in patients in a tertiary care hospital, South India: Underlying risk factors in $\mathrm{TB}$ patients,” Trop. Med. Int. Health, vol. 16, no. 1, pp. 74-78, Jan. 2011.

[21] Public Health Agency of Canada, The Chief public health officer's report on the state of public health in Canada, 2013: Infectious disease-The neverending threat. Ottawa: Public Health Agency of Canada, Government of Canada; 2013.

[22] J.-P. Millet et al., “Tuberculosis recurrence after completion treatment in a European city: reinfection or relapse?,” PLoS ONE, vol. 8, no. 6, p. e64898, 2013.

[23] World Lung Foundation, Directions-the deadly paring of tuberculosis and smoking. New York: World Lung Foundation, 2, no. 2; 2011.

[24] J.-Y. Feng et al., "Impact of cigarette smoking on latent tuberculosis infection: does age matter?,” Eur. Respir. J., vol. 43, no. 2, pp. 630-632, Jan. 2014.

[25] M. Gegia et al., “Tobacco smoking and tuberculosis treatment outcomes: a prospective cohort study in Georgia,” Bull. World Health Organization, vol. 93, no. 6, pp. 390-399, Jun. 2015.
[26] Jianming Wang, Hongbing Shen, "Review of cigarette smoking and tuberculosis in China: intervention is needed for smoking cessation among tuberculosis patients," BMC Public Health, vol. 9:292, 2009.

[27] K. E. Dooley and R. E. Chaisson, "Tuberculosis and diabetes mellitus: convergence of two epidemics," Lancet Infect. Dis., vol. 9, no. 12, pp. 737-746, Dec. 2009.

[28] P. Baghaei, M. Marjani, P. Javanmard, P. Tabarsi, and M.

R. Masjedi, "Diabetes mellitus and tuberculosis facts and controversies," J Diabetes Metab Disord, vol. 12, no. 1, p. 58, 2013.

[29] C. Lienhardt12, "From exposure to disease: the role of environmental factors in susceptibility to and development of tuberculosis,” Epidemiol Rev, vol. 23, no. 2, 2001.

[30] C. R. MacIntyre, N. Kendig, L. Kummer, S. Birago, and N. M. Graham, "Impact of tuberculosis control measures and crowding on the incidence of tuberculosis infection in Maryland prisons," Clin. Infect. Dis., vol. 24, no. 6, pp. 1060-1067, 1997. 